\title{
Usage de la tomodensitométrie pour le diagnostic de la maladie coronarienne
}

\author{
Conseil d'experts du \\ Swiss Medical Board*: \\ Eva Cignacco ${ }^{a}$, \\ Peter Jüni ${ }^{b}$, Peter Meier-Abt ${ }^{c}$, \\ Urs Metzgerd, Nikola Biller- \\ Andorno ${ }^{e}$, Stefan Felderf, \\ Brigitte Tagg \\ a Institut des sciences \\ infirmières, Université \\ de Bâle (Médecine) \\ b Prof. d'épidémiologie \\ clinique, Université de Berne \\ (Médecine) \\ c Prof. em. de pharmacologie \\ clinique, vicerecteur \\ de l'Université de Bâle \\ (Médecine) \\ d Prof. ém. médecin chef en \\ chirurgie, Zurich (Médecine) \\ e Prof. d'éthique biomédicale, \\ Université de Zurich (Ethique) \\ f Prof. d'économie de la santé, \\ Université de Bâle \\ g Prof. de droit pénal, procé- \\ dure pénale et droit \\ de la médecine, \\ Université de Zurich (Droit)
}

* Organisation et composition sous www.medical-board.ch/ index.php?id=818\&L=1

\section{Correspondance:}

Susanna Marti Calmell

Secrétariat Swiss Medical Board Obstgartenstrasse 21

CH-8090 Zurich

Tél. 0432592479

info[at]medical-board

www.swissmedicalboard.ch
Quel est le rendement diagnostique d'une tomodensitométrie cardiaque utilisant des appareils de nouvelle génération (New Generation Cardiac Computed Tomography/ NGCCT) par rapport à la coronarographie invasive habituellement pratiquée? Le conseil d'experts du Swiss Medical Board s'est penché sur cette question dans le rapport qu'il vient de publier. Pour établir l'évidence clinique, il s'est basé sur trois documents du National Institute of Health and Clinical Excellence (NICE) et a conclu que, chez certains groupes d'individus, la tomodensitométrie cardiaque est indiquée pour le diagnostic d'une maladie coronarienne.

\section{Résumé du rapport original}

On entend par «maladie coronarienne» les lésions des artères qui alimentent le muscle cardiaque en sang (vaisseaux ou artères coronaires). Il s'agit là d'une diminution croissante du diamètre des artères pouvant indirectement entraîner des complications aigües potentiellement fatales (infarctus du myocarde, par exemple). En Suisse, 8800 personnes meurent chaque année d'une maladie coronarienne. Ceci correspond à 14,5\% du nombre total de décès. Pour détecter une maladie coronarienne, on dispose entre autres de deux techniques d'imagerie médicale: la tomodensitométrie cardiaque et la coronarographie invasive.

Le présent rapport du Swiss Medical Board se penche sur la question de savoir comment se compare le rendement diagnostique d'une tomodensitométrie cardiaque utilisant des appareils de la nouvelle génération (New Generation Cardiac Computed Tomography, NGCCT) par rapport à la coronarographie invasive (gold standard). Sont en outre examinés les effets négatifs potentiels des deux techniques et le rapport coût-efficacité. Le présent rapport se fonde sur trois documents du National Institute of Health and Clinical Excellence (NICE) de Grande-Bretagne, ainsi que sur des réflexions complémentaires du Swiss Medical Board.

Sur la base de ces fondements, on peut conclure que la tomodensitométrie cardiaque peut remplacer sans inconvénient la coronarographie invasive pour le diagnostic d'une maladie coronarienne soupçonnée chez certains groupes d'individus. Cela dépendra de la «probabilité pré-test», c'est-à-dire la probabilité estimée, sans examen plus approfondi, qu'un individu soit atteint d'une maladie coronarienne. Chez certains groupes d'individus, le rapport coût-effica- cité de la coronarographie invasive est relativement défavorable. Les connaissances tirées de la présente analyse conduisent aux recommandations suivantes:

- Avant d'utiliser une technique d'imagerie médicale chez un patient soupçonné d'être atteint d'une maladie coronarienne, il convient au préalable d'évaluer soigneusement la probabilité prétest.

- En cas de probabilité pré-test faible (généralement inférieure à $10 \%$ ), l'utilisation d'une technique d'imagerie médicale n'est pas indiquée.

- Chez les individus chez lesquels la probabilité pré-test a été évaluée à entre $10 \%$ et $30 \%$ environ, il convient, pour le diagnostic, de réaliser une tomodensitométrie cardiaque à l'aide d'appareils de la nouvelle génération (NGCCT), dans la mesure où l'imagerie médicale est indispensable. La réalisation d'emblée d'une coronarographie invasive est contre-indiquée chez ce groupe d'individus. La question de savoir si, en cas de résultats positifs, une coronarographie invasive doit ensuite être réalisée dépendra des résultats de la tomodensitométrie et de chaque cas.

- En cas de probabilité pré-test plus élevée (généralement entre $30 \%$ et $50 \%$ environ), il convient de réaliser, comme première méthode d'imagerie, une tomodensitométrie cardiaque. En cas de résultats positifs avec des appareils NGCCT, il faudra, le cas échéant, explorer davantage par coronarographie invasive et appliquer éventuellement un traitement.

- Les prestataires doivent expliquer aux patients les différentes techniques, même si une de ces techniques ne peut pas être proposée dans leur propre établissement.

\section{Thèmes traités par le conseil d'experts en 2013}

Pour l'élaboration de rapports HTA, les deux thèmes suivants ont été choisis dans un premier temps pour l'année 2013, conformément à la procédure normale (input des personnes intéressées, évaluation en fonction d'un cadre de priorisation, décision de l'organisation responsable): «Dépistage systématique par mammographie» et «Traitement par statines comme prévention primaire de maladies cardiovasculaires». Informations détaillées sous: www.swissmedicalboard.ch 